\title{
Self-Assembled 2D Protein Crystals as Templates for Ordered Metallic Nano- Arrays
}

\author{
R. Mogul ${ }^{1}$, R. A. McMillan², C. D. Paavola ${ }^{2}$, J. D. Trent ${ }^{2}$, N. J. Zaluzec ${ }^{3}$ \\ 1- Education Associates, Mail Stop 239-15, Moffett Field, CA 94035, USA \\ 2- NASA Ames Research Center, Center for Nanotechnology and Astrobiology Technology Branch, \\ Mail Stop 239-15, Moffett Field, CA 94035, USA \\ 3- Argonne National Laboratory, Materials Science Division, Argonne, Il 60440, USA
}

The arrangement of inorganic materials into nanoscale ordered arrays holds great promise for the production of new types of electronic, magnetic, and photonic devices. Fabricating ordered arrays on the nanoscale exceeds the limits of traditional lithographic techniques; therefore, new techniques, including those involving biomolecules such as proteins, are under investigation. We are using the heat shock protein (HSP60) from Sulfolobus shibatae, which self-assembles into ring structures known as chaperonins, to make ordered two-dimensional inorganic arrays. Chaperonins can be induced to form higher-order two-dimensional crystals, which can be genetically engineered into nanoscale templates that bind inorganic materials. We genetically inserted cysteine residues into the HSP60s to form two-dimensional arrays of chaperonins possessing reactive thiol groups that bind gold or platinum. Ordered arrays were created using two methods: (1) by covalent attachment of gold nanoparticles (1.4 nm Nanogold) to HSP60s prior to crystallization into arrays (Fig. 1; also see Ref. 1) and (2) by electroless deposition of $\mathrm{NiSO}_{4}$ (Fig. 2) onto platinum-activated two-dimensional crystals.

Genetically engineered chaperonins containing cysteine mutations were designed, expressed, and purified as described by McMillan et. al. Two-dimensional crystals of chaperonins were made with concentrated HSP60 $(1.5 \mathrm{mg} / \mathrm{ml})$ in the presence of $4 \mathrm{mM} \mathrm{ATP}, 10 \mathrm{mM} \mathrm{MgCl}_{2}$, and $0.01 \% \mathrm{NaN}_{3}$ in $25 \mathrm{mM}$ HEPES ( $\mathrm{pH} \mathrm{7.5)}$ ) and incubated for $\sim 12 \mathrm{hr}$ at $4^{\circ} \mathrm{C}$. Using a 1:10 dilution of the crystal suspension, the crystals were immobilized on $\mathrm{SiO}$ or formvar coated copper TEM grids (200 mesh). Grids were floated on the suspension for $30 \mathrm{~s}$ and washed in water for $30 \mathrm{~s}$. The immobilized crystals on the grids were activated in $6 \mathrm{mM} \mathrm{K}_{2} \mathrm{PtCl}_{4}$ for $2 \mathrm{~min}$ followed by two $30 \mathrm{~s}$ washes in 25 $\mathrm{mM} \mathrm{NaOAc}\left(\mathrm{pH} \mathrm{4.5)}\right.$. They were then metalized in a solution containing $25 \mathrm{mM} \mathrm{NiSO}_{4}$ and $5 \mathrm{~g} / \mathrm{L}$ dimethylamine borane complex (DMAB) for $30 \mathrm{~s}$ (Ref. 2). Reactions were immediately quenched by two $30 \mathrm{~s}$ water washes and the grids dried under vacuum.

Conventional TEM imaging was conducted on a LEO $912 \mathrm{AB}$ at $60 \mathrm{kV}$ at NASA Ames, while high resolution nanoscale imaging and characterization was conducted on a FEI Tecnai F20 operating at $200 \mathrm{kV}$ at ANL. Images were recorded using STEM HAADF imaging mode, while elemental characterization of all samples was confirmed using X-ray Energy Dispersive and Electron Energy Loss Spectroscopy. Control experiments conducted without exposure to the metal salts resulted in 2D crystals without ordered metallic arrays.

We observed that the Nanogold particles covalently bound to the chaperonin template were hexagonally packed and aggregated in the cores of the chaperonins (Fig. 1). In contrast, the electroless deposition resulted in a non-uniform distribution of nickel on the chaperonin surface with regions of high density that yield the hexagonally packed array (Fig. 2).

These results demonstrate that genetically modified chaperonins can direct the formation and ordering of inorganic arrays of preformed metal particles or their precursors. 


\section{References}

[1] R. A. McMillan et al., Nature Materials 1 (2002) 247.

[2] M. Mertig, Thin Solid Films 305 (1997) 248.

[3] This work was supported by the NASA Ames Center for Nanotechnology, DoE, DARPA, and by grants at ANL from DoE under contract BES-MS W-31-109-Eng-38.
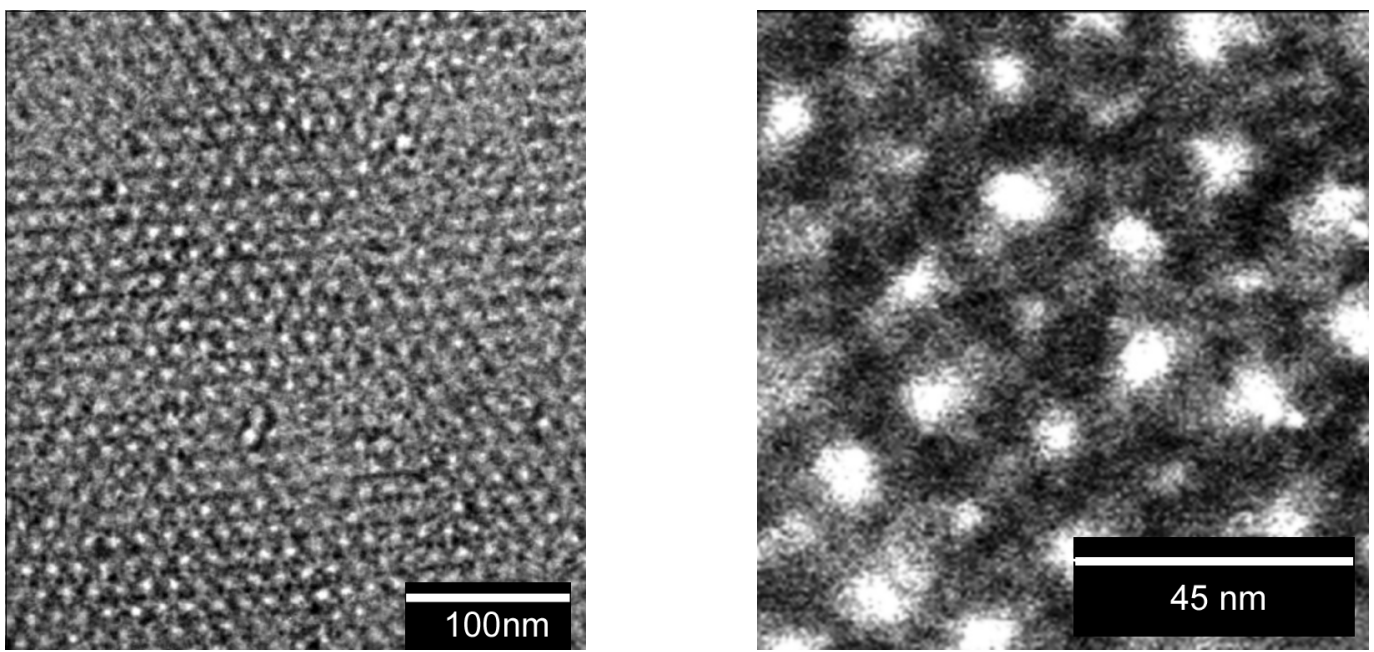

Fig. 1. Nanoscale ordered Au particles on S. shibatae chaperonin protein template
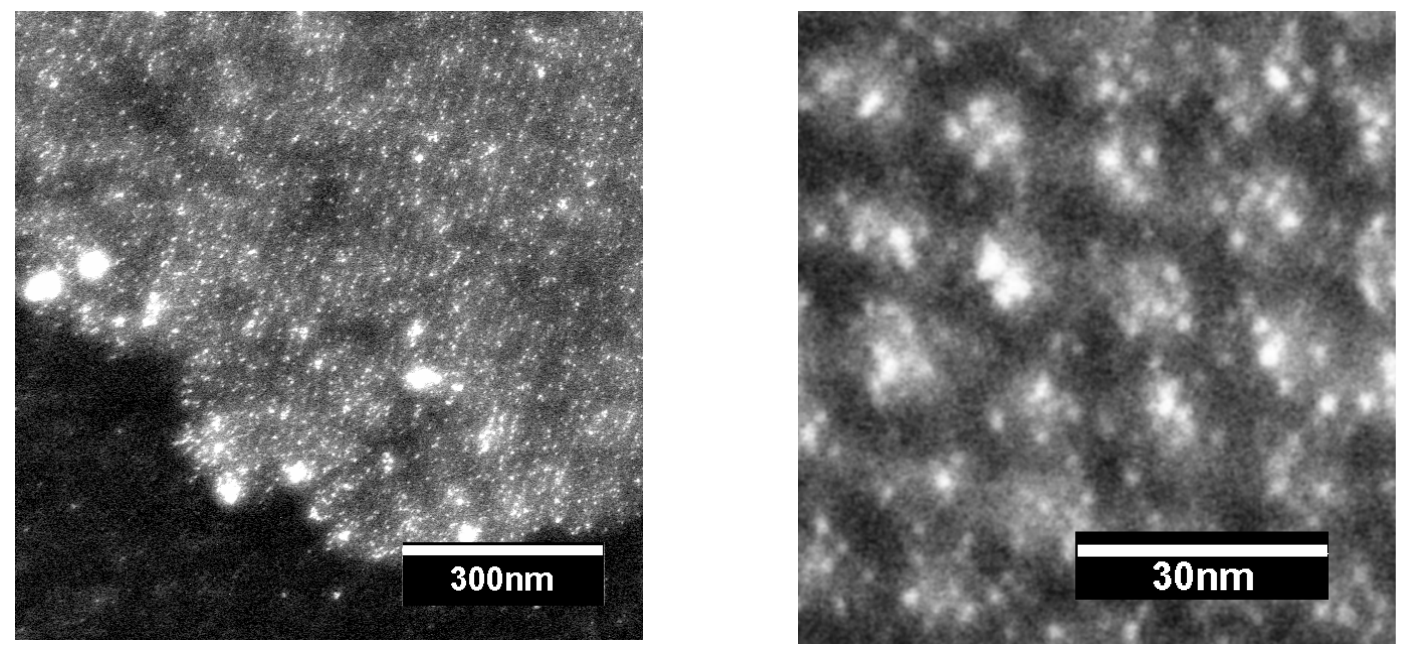

Fig. 2. Nanoscale ordered Ni particles on engineered S. shibatae chaperonin protein template 